\title{
INTEGRAL SPECTROSCOPY OF IRAS 17208-0014: IMPLICATIONS FOR THE EVOLUTIONARY SCENARIOS OF ULTRALUMINOUS INFRARED GALAXIES ${ }^{1}$
}

\author{
Santiago ARribas ${ }^{2,3}$ and Luis Colina ${ }^{4}$ \\ Received 2003 February 12; accepted 2003 March 19
}

\begin{abstract}
New integral field optical fiber spectroscopy obtained with the INTEGRAL system, together with archival HST WFPC2 and NICMOS images, has been used to investigate the ultraluminous infrared galaxy (ULIRG) IRAS 17208-0014, one of the coldest and most luminous objects in the IRAS 1 Jy sample. We have found that the optical nucleus is not coincident with the true (near-IR and dynamical) nucleus, but that it is displaced by $1.3 \mathrm{kpc}(1 . .5)$ from it. As a consequence, the previous optical spectral classifications for the nucleus of this galaxy have to be changed from $\mathrm{H}$ II to LINER. The ionized gas emission is concentrated around the optical nucleus, where a young (5-6 Myr), massive $\left[(3 \pm 1) \times 10^{8} M_{\odot}\right]$, and luminous $\left[(6 \pm 2) \times 10^{10} L_{\odot}\right]$ starburst is detected. Contrary to what is found in dynamically young ULIRGs, no strong line emission tracing star-forming regions, or tidal dwarf galaxies, is detected in the inner parts of the tidal tails. The two-dimensional gas velocity field identifies the optically faint $K$-band nucleus as the dynamical nucleus of the galaxy and shows that the $3 \mathrm{kpc}$, tilted $\left(i \sim 35^{\circ}\right)$ disk is rotating at $\Delta v \sin i=250 \mathrm{~km} \mathrm{~s}^{-1}$. Radial motions of gas are found along the minor kinematic axis, which, according to the geometry of the system, are well interpreted as inflows perpendicular to the inner disk. The existence of such inflows supports the idea that, as a consequence of the merging process, gas is channeling from the external regions, several kiloparsecs away, into the nuclear regions where the massive starburst reported above is taking place. The kinematical, morphological, and photometric evidence presented here supports the idea that in IRAS 17208-0014 we are witnessing a luminous, cool ULIRG that is at the final coalescence phase of a system composed of two spiral galaxies with $m \leq m^{*}$ and a mass ratio of $\sim 2: 1$, each consisting of a disk + bulge internal structure, that have been involved in a prograde encounter. This system will most likely evolve into an intermediate-mass $\left(\sim L^{*}\right)$ elliptical galaxy. The multifrequency empirical evidence gathered so far shows no trace of a luminous QSO and indicates that starbursts dominate the energy output in this galaxy. Therefore, IRAS 17208-0014 does not follow the behavior expected in the "ULIRG-to-QSO" evolutionary scenario proposed by Sanders et al., but it supports the one recently proposed by Colina et al., in which two low-mass disk galaxies would produce luminous, cool ULIRGs that would not evolve into QSOs. The present study illustrates some caveats to bear in mind when studying high- $z$ galaxies lacking two-dimensional spectral information of adequate linear resolution and shows that near- and mid-IR integral field spectroscopy is needed to derive the relevant astrophysical quantities.
\end{abstract}

Subject headings: galaxies: active — galaxies: individual (IRAS 17208-0014) — galaxies: interactions — galaxies: nuclei — galaxies: starburst

\section{INTRODUCTION}

Ultraluminous infrared galaxies (ULIRGs) are the most luminous objects in the local universe, with bolometric luminosities $L_{\mathrm{bol}} \approx L_{\mathrm{IR}} \geq 10^{12} L_{\odot}$. Having large amounts of gas and dust, they are undergoing intense starburst activity triggered by interactions and/or mergers. This starburst activity is believed to be their major energy reservoir (Genzel et al. 1998), although the relative importance of the

\footnotetext{
${ }^{1}$ Based on observations with the William Herschel Telescope, operated on the island of La Palma by the ING in the Spanish Observatorio del Roque de los Muchachos of the Instituto de Astrofísica de Canarias. Based also on observations with the NASA/ESA Hubble Space Telescope (HST), obtained at the Space Telescope Science Institute, which is operated by the Association of Universities for Research in Astronomy (AURA), Inc., under NASA contract NAS 5-26555.

${ }^{2}$ Space Telescope Science Institute, 3700 San Martin Drive, Baltimore, MD 21218; arribas@stsci.edu. Also affiliated with the Space Telescope Division, Research and Science Support Department of ESA.

${ }^{3}$ On leave from the Instituto de Astrofísica de Canarias, Consejo Superior de Investigaciones Científicas (CSIC).

${ }^{4}$ Instituto de Estructura de la Materia, Consejo Superior de Investigaciones Científicas (CSIC), Serrano 121, 28006 Madrid, Spain; colina@isis.iem.csic.es.
}

active galactic nucleus (AGN) phenomenon as the source of energy in ULIRGs is under debate (see, e.g., Sanders \& Mirabel 1996). Sanders et al. (1988) suggested an evolutionary sequence according to which ULIRGs are the precursors of optical quasars. In this scenario, warm ULIRGs (i.e., $\left.f_{25 \mu \mathrm{m}} / f_{60 \mu \mathrm{m}}>0.2\right)$ are in a later evolutionary stage than cool ULIRGs $\left(f_{25 \mu \mathrm{m}} / f_{60 \mu \mathrm{m}}<0.2\right)$. High spatial resolution imaging and nuclear spectroscopy have found evidence in support of this scenario (see, e.g., Surace et al. 1998; Veilleux, Kim, \& Sanders 1999, 2002; Kim, Veilleux, \& Sanders 2002 and references therein). On the other hand, Genzel et al. (2001) have found that most ULIRGs seem to be evolving toward intermediate-mass $\left(L^{*}\right)$ elliptical galaxies, suggesting that ULIRG mergers are ellipticals in formation. However, details on how the ULIRGs evolve remain very uncertain, despite the progress made recently in characterizing merger evolution on the basis of theoretical models (see Mihos \& Hernquist 1996; Naab \& Burkert 2001 and references therein). These studies show that gasdynamics and star formation in mergers are closely related. They also indicate that the internal structure of the merging galaxies and their mass ratio are key factors in determining the characteristics of ULIRGs. 
From an observational point of view, detailed studies of the complex kinematics and ionization structures of these objects demand the analysis of their spectral properties in two dimensions (see also Murphy et al. 2001). However, only a very limited number of this type of studies exist so far (e.g., Mihos \& Bothun 1998; Wilman, Crawford, \& Abraham 1999). We have recently started a program aimed at studying a representative sample of ULIRGs combining integral field spectroscopy (IFS) and high-resolution HST imaging. Some of the main results obtained so far for other ULIRGs, such as Mrk 273, IRAS 12112+0305, IRAS $08572+3915$, Arp 220, and IRAS $15206+3342$, can be found elsewhere (see Arribas \& Colina 2002 and references therein).

In this paper, we present new INTEGRAL spectroscopy and archival HST imaging of IRAS 17208-0014. This is a ULIRG with some interesting properties. Despite being the second most luminous galaxy in the sample studied by Kim et al. $(1995)\left(L_{\mathrm{bol}}=10^{12.4} L_{\odot}\right)$, it does not show evidence of an AGN. In particular, (1) it has been reported as a case of an $\mathrm{H}$ II/starburst excitation in the optical (Veilleux et al. 1995) and in the mid-infrared (Lutz, Veilleux, \& Genzel 1999), (2) the nucleus in the near- and mid-infrared images is extended ( $\sim 2^{\prime \prime}$; Scoville et al. 2000; Soifer et al. 2000), and (3) the near-infrared search for a hidden broad line indicated no signs of a BLR (Goldader et al. 1995). HST NICMOS observations (Scoville et al. 2000) have revealed the presence of at least 18 clusters of star formation, mainly concentrated in the inner regions, where extinction is high. The presence of a rotating disk of molecular gas has been inferred from CO observations (Downes \& Solomon 1998). The nucleus also has one of the strongest known $\mathrm{OH}$ megamasers and a radio continuum source of $\sim 250 \mathrm{pc}$ in size (Martin et al. 1989).

Ground-based optical imaging of IRAS 17208-0014 (Murphy et al. 1996; Duc, Mirabel, \& Maza 1997) has revealed a disturbed and irregular system, with two prominent tidal tails toward the southeast and northwest extending to distances larger than $20 \mathrm{kpc}$. This morphology, together with the fact that near-infrared images show a luminosity profile typical of ellipticals, has led to the suggestion that this is a case in which an elliptical galaxy is being formed after a disk-disk collision (Duc et al. 1997). Furthermore, IRAS $17208-0014$ is located near the fundamental plane (FP), in a region populated by fast-rotating elliptical and lenticular galaxies with intermediate mass and disky isophotes (Genzel et al. 2001). This result also suggests that the merger is in a relatively advanced phase and will eventually lead to an elliptical galaxy with the above-mentioned characteristics.

The new optical INTEGRAL spectroscopy and $H S T$ imaging presented here $(\S 2)$ are used to characterize the stellar structure $(\S 3.1)$, the ionized gas morphology and excitation $(\S 3.2)$, the properties of the extranuclear starburst (§ 3.3), and the two-dimensional kinematics of the ionized gas $(\S 3.4)$ in this galaxy. On the basis of these results and previously published studies, we discuss in $\S 4$ the properties and evolutionary phase of the merging system, as well as the implications for studies of high- $z$ galaxies. Finally, in $\S 5$, the main results are summarized.

At the assumed distance of $183 \mathrm{Mpc}$ for IRAS 17202-0014, $1^{\prime \prime}$ corresponds to a linear size of $888 \mathrm{pc}$. Throughout the paper, a Hubble constant of $70 \mathrm{~km} \mathrm{~s}^{-1}$ $\mathrm{Mpc}^{-1}$ is assumed.

\section{OBSERVATIONS AND DATA REDUCTION}

Integral field spectroscopy of IRAS 17202-0014 was obtained with the INTEGRAL system (Arribas et al. 1998) connected to WYFFOS (Wide-Field Fiber Optic Spectrograph; Bingham et al. 1994) on the $4.2 \mathrm{~m}$ William Herschel Telescope during 1999 April 3. The bundle of fibers SB2 (standard bundle number 2), consisting of 219 fibers, each $0 "$.9 in diameter, was used. This is arranged such that 189 fibers cover a rectangular area of $16^{\prime \prime} .5 \times 12^{\prime \prime} .3(14.6 \times 10.9$ $\mathrm{kpc}$ at the distance of IRAS 17202-0014), while 30 additional fibers, forming a ring of $90^{\prime \prime}$ in diameter concentric with the rectangle, simultaneously measure the sky background (see Arribas \& Mediavilla 2000 for further details on integral field fiber spectroscopy). The spectra were taken using a 600 lines $\mathrm{mm}^{-1}$ grating with an effective resolution of $4.8 \AA$ and covering the $5000-7900 \AA$ spectral range. The total integration time was $7800 \mathrm{~s}$ split into four separate exposures, with seeing of about 1 ". 1 .

Data reduction followed the standard procedures applied to spectra obtained with two-dimensional fiber spectrographs (see, for instance, Arribas \& Mediavilla 2000 and references therein). As an example, the two-dimensional distribution of the reduced spectra in the range of the redshifted $\mathrm{H} \alpha+[\mathrm{N}$ II $]+[\mathrm{S}$ II] lines (i.e., 6792-7045 $\AA$ ) are presented in Figure 1. $\mathrm{H} \beta$ and [O III] $\lambda \lambda 4959,5007$ appeared conspicuously in only four spectra: 102, 103, 104, and 107 (not shown).

For each spectrum, the radial velocities and velocity dispersions were measured by adjusting Gaussian functions to the observed emission-line profiles using the DIPSO package (Howarth \& Murray 1988). The uncertainties associated with the individual velocities are estimated to be on the order of $\pm 15 \mathrm{~km} \mathrm{~s}^{-1}$. The velocity dispersion values presented in this paper are corrected for instrumental profile and redshift.

The archival HST images of IRAS 17208-0014 obtained in the $I$ band (F814W filter, WFPC2) and $K$ band (F222M, NIC2) were originally taken as part of programs 6346 (PI: K. Borne) and 7116 (PI: N. Scoville), respectively. The total exposure time for the F814W image was $800 \mathrm{~s}$, divided into two identical exposures of $400 \mathrm{~s}$ each using the CR-SPLIT option. The data were recalibrated at the time of dearchiving using on-the-fly calibration software and the best reference calibration files available. The NICMOS F222M image, first analyzed and published in Scoville et al. (2000), with a total integration time of $288 \mathrm{~s}$, was recalibrated in 2002 October using calnica and calnicb and the best available reference files.

\section{RESULTS}

\subsection{Stellar Structure and Differential Extinction Effects}

The INTEGRAL red continuum image, taken with a narrow 7029-7068 ̊ window (Fig. 2, bottom left-hand panel), has a good morphological resemblance to the WFPC2 I-band image (Fig. 2, top left-hand panel). This allows us to establish the absolute positioning of the INTEGRAL maps with an uncertainty of about 0 ". 3 with respect to the $H S T$ reference frame. In addition, the WFPC2 and NICMOS images could be placed on the same coordinate system using pointlike structures present in both images.

The morphology of the outer low surface brightness region in the red continuum images is like that of a per- 


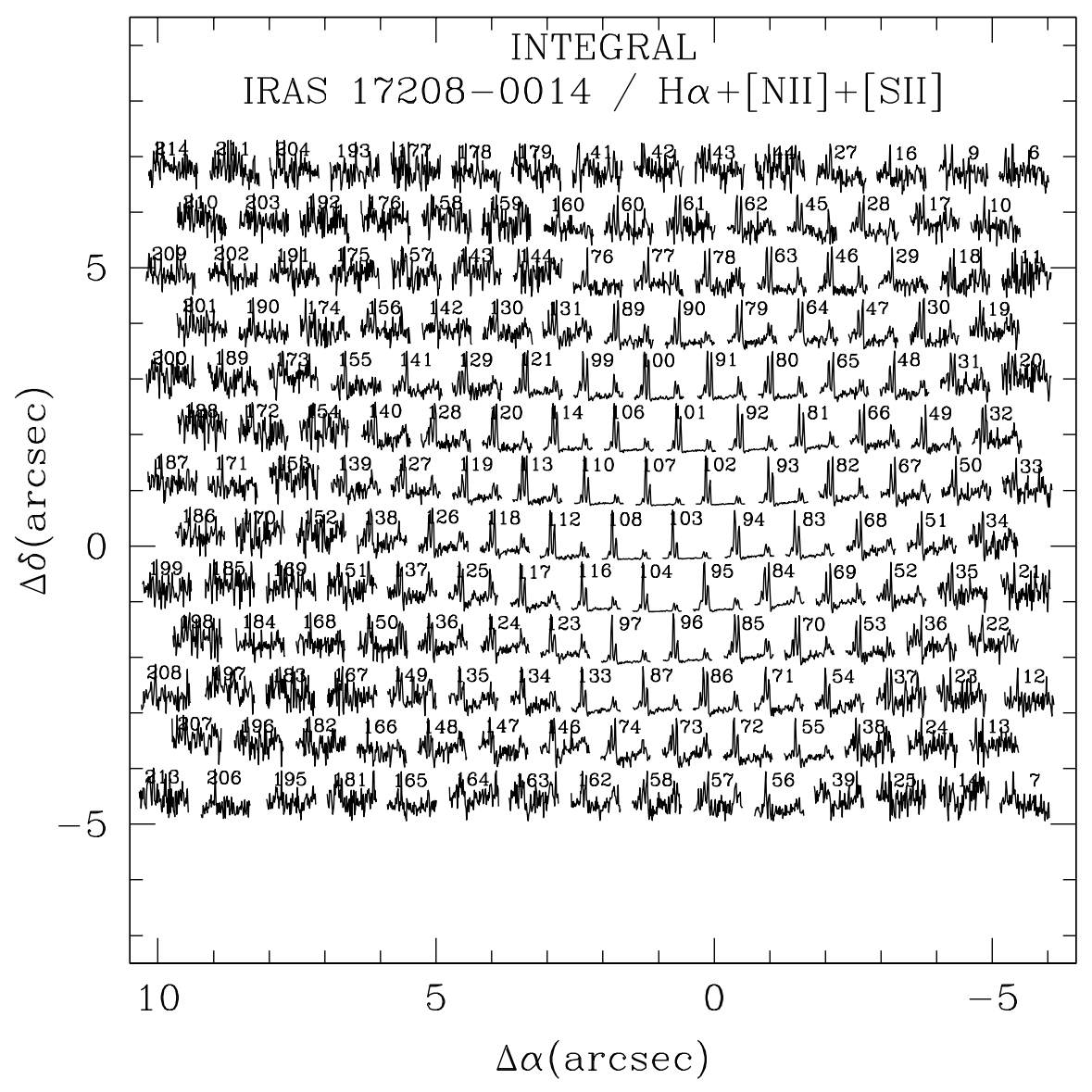

FIG. 1.-Spatial distribution of the spectra in the spectral range 6792-7045 $\AA$ corresponding to the redshifted $\mathrm{H} \alpha+[\mathrm{N}$ II] emission-line complex. Numbers indicate the fiber/spectrum position at the slit/detector. These spectra are linearly scaled between their lowest and highest values in the represented spectral range.

turbed elliptical galaxy. However, inward, the presence of spiral arm-like structures is rather conspicuous. The innermost star-forming clusters, previously identified in the nearIR with NICMOS (Scoville et al. 2000; see also Fig. 3), are also detected in the WFPC2 I-band image. Leaving aside the contribution of the circumnuclear clusters, the inner isophotes in the infrared images define an elliptical structure, suggesting the presence of a tilted disk with $i=35^{\circ} \pm 15^{\circ}$ and $3 \pm 0.5 \mathrm{kpc}$ in diameter (Fig. 3 ).

There is a general consensus that ULIRGs are galaxies where most of the active regions, either massive starbursts or AGNs, are enshrouded in dust. However, the dust distribution, although concentrated toward the nuclear regions, i.e., the inner few kiloparsecs, is very patchy, and therefore differential extinction effects play a major role in interpreting the results (see, e.g., Colina et al. 2001). In IRAS 17208-0014, the most relevant effect of the differential extinction is that the true nucleus of the galaxy does not coincide with the region identified as the nucleus in the optical, but is located about $1.15(1.3 \mathrm{kpc})$ southwest of it (compare top left-hand panel of Fig. 2 with Fig. 3). In the direction of the optical nucleus, the hydrogen recombination lines (see $\S 3.2$ ) in the INTEGRAL spectra indicate an extinction $A_{V}$ of about $5.5 \mathrm{mag}$, in good agreement with previous measurements (see Table 1). No $\mathrm{H} \beta$ is detected toward the near-IR nucleus. However, assuming that extinction is in a foreground screen (i.e., not mixed with the stars) and using the law derived by Rieke \& Lebofsky
(1985), an extinction of up to $A_{V}=8 \mathrm{mag}$ is inferred from the infrared colors (Scoville et al. 2000). However, this could be a lower limit. Scoville et al. pointed out that the nuclear power source in IRAS 17208-0014 could be buried even at near-IR wavelengths.

A secondary emission peak in the optical, located at about $3^{\prime \prime}(2.7 \mathrm{kpc})$ southeast of the optical nucleus, is rather clear in both the INTEGRAL and the WFPC2 images. This region could, in principle, be identified as the second nucleus of a merging pair. However, its flux loses strength at longer infrared wavelengths and nearly disappears in the $2.2 \mu \mathrm{m}$ map (Fig. 3), suggesting that its optical emission is associated with a relatively young population located on one of the spiral arm-like structures.

\subsection{Ionized Gas Morphology and Excitation Structure}

The structure of the warm ionized gas is traced here by the $\mathrm{H} \alpha$ map (Fig. 2, top middle panel). This image shows a bright nucleus and a low-intensity extended emission of at least $8 \mathrm{kpc}$, elongated in the southeast-northwest direction. The structure of the outer envelope is reminiscent of that of the stellar component, although it does not have a one-toone correspondence. In fact, the ionized gas emission is more compact, and it extends toward the north, where a tidal tail is observed in deep optical continuum images (see Murphy et al. 1996; Duc et al. 1997). However, no strong line emission tracing star-forming regions or tidal dwarf 

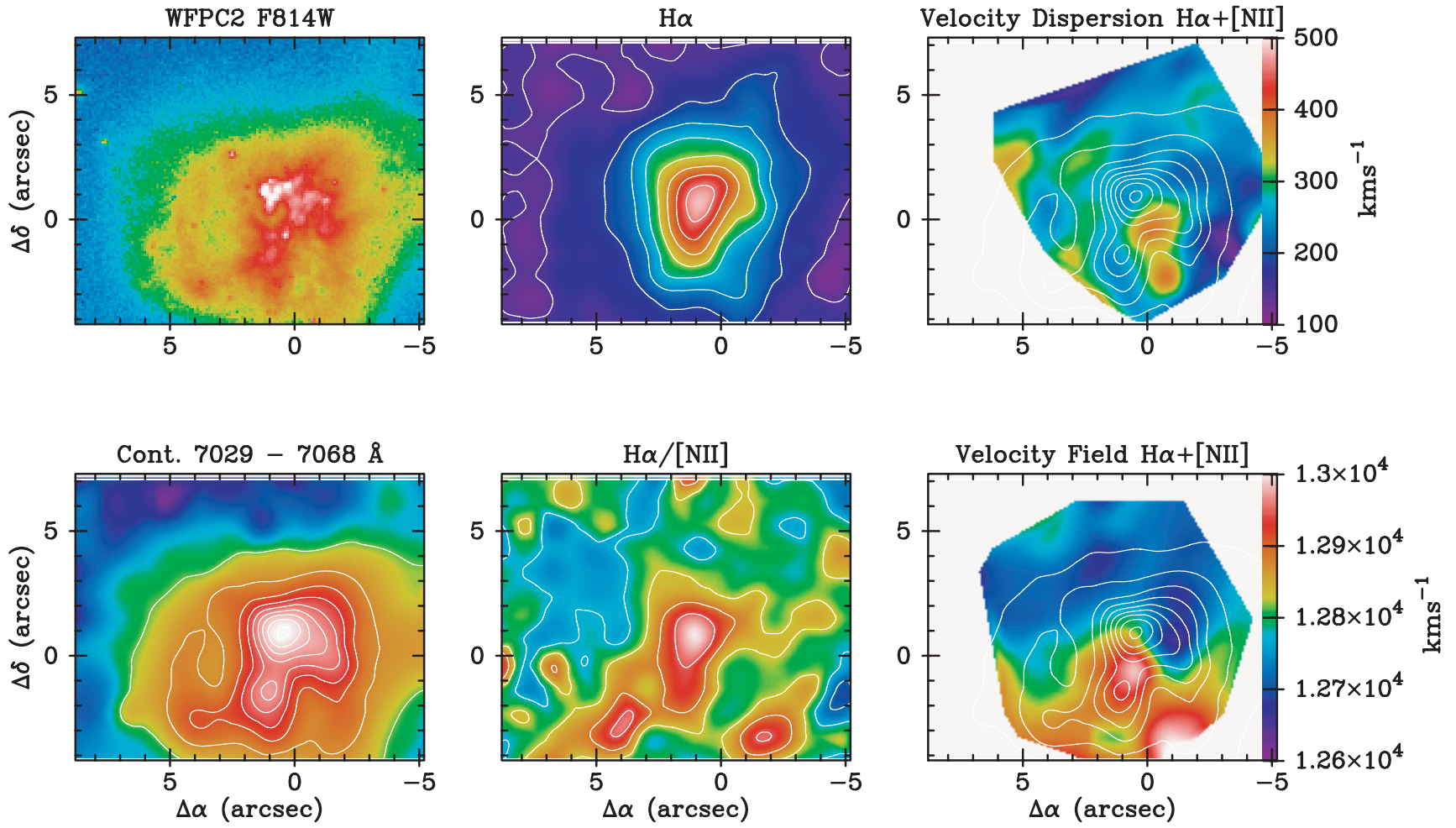

FIG. 2.-INTEGRAL and HST WFPC2 images in the central regions of IRAS 17208-0014. Values for the isolines (in arbitrary units) are, in the H $\alpha$ map (top middle panel), 2.024, 5.402, 11.04, 20.43, 36.11, 62.26, 105.9, and 178.6; in the continuum map (bottom left-hand panel), 0.667, 1.333, 2.00, 2.667, 3.333, $4.00,4.667,5.334$, and 6.00 (contours in the right-hand panels correspond to the same intensity levels); and in the $\mathrm{H} \alpha /[\mathrm{N}$ II] map (bottom middle panel), 0.443 (blue), 0.914, 1.386, 1.857, and 2.328 (white). For the velocity maps (right-hand panels), the scale is indicated at the right of each panel. North is at the top, and east is to the left.

galaxies is observed in the inner parts of the tidal tails, as found in some double-nucleus ULIRGs (e.g., IRAS 12112+0305: Colina et al. 2000; IRAS 08572+3915: Arribas, Colina, \& Borne 2000; IRAS 14348-1447: A. Monreal et al. 2003, in preparation).

The ionized gas in the optical nucleus exhibits excitation conditions characteristic of $\mathrm{H}$ II regions (see Table 1 and
Fig. 2). Strong $\mathrm{H} \alpha$, relative to [N II], is also detected toward the south of the optical nucleus. However, apart from these regions, the [N II] emission is at least as intense as $\mathrm{H} \alpha$ in the rest of the regions (see Fig. 1). Further support for this dichotomy in the excitation conditions comes from the ratio [S II] $/ \mathrm{H} \alpha$, which has a minimum in the optical nucleus and is considerably higher elsewhere (see Fig. 1 and compare,

TABLE 1

Ionized Gas Properties of the Optical and NeAR-IR NUCle

\begin{tabular}{|c|c|c|c|c|c|c|c|c|}
\hline $\begin{array}{l}\text { Region } \\
\text { (1) }\end{array}$ & $\begin{array}{c}A_{V}{ }^{\mathrm{a}} \\
(\mathrm{mag}) \\
(2)\end{array}$ & $\begin{array}{c}F(\mathrm{H} \alpha)^{\mathrm{b}} \\
\left(\times 10^{-14} \mathrm{ergs}\right. \\
\left.\mathrm{s}^{-1} \mathrm{~cm}^{-2}\right) \\
(3)\end{array}$ & $\begin{array}{c}\mathrm{EW}(\mathrm{H} \alpha)^{\mathrm{c}} \\
(\AA) \\
(4)\end{array}$ & $\begin{array}{c}\log L(\mathrm{H} \alpha)^{\mathrm{d}} \\
\left(L_{\odot}\right) \\
(5)\end{array}$ & $\begin{array}{c}\log \left(\left[\mathrm{O}_{\mathrm{III}}\right] / \mathrm{H} \beta\right)^{\mathrm{e}} \\
(6)\end{array}$ & $\begin{array}{c}\log ([\mathrm{O} \mathrm{I}] / \mathrm{H} \alpha)^{\mathrm{e}} \\
(7)\end{array}$ & 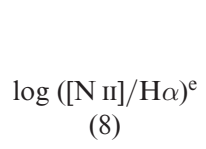 & $\begin{array}{c}\log ([\mathrm{S} \mathrm{II}] / \mathrm{H} \alpha)^{\mathrm{c}} \\
(9)\end{array}$ \\
\hline Optical nucleus .... & $5.5 \pm 0.5$ & $0.56 \pm 0.1$ & $94 \pm 15$ & $8.55 \pm 0.1$ & $\begin{array}{l}-0.002 \pm 0.09 \\
-0.10 \pm 0.09\end{array}$ & $\begin{array}{l}-1.60 \pm 0.1 \\
-1.35 \pm 0.1\end{array}$ & $\begin{array}{l}-0.40 \pm 0.06 \\
-0.41 \pm 0.06\end{array}$ & $\begin{array}{l}-0.51 \pm 0.06 \\
-0.56 \pm 0.06\end{array}$ \\
\hline Optical nucleus ${ }^{\mathrm{f}} \ldots$. & 5.4 & 1.79 & 51 & 9.05 & -0.16 & -1.22 & -0.35 & -0.54 \\
\hline
\end{tabular}

a Internal extinction derived from the $\mathrm{H} \alpha / \mathrm{H} \beta$ ratios for the optical nucleus and from the near-IR colors for the infrared nucleus.

${ }^{b}$ Observed $\mathrm{H} \alpha$ flux, not corrected for extinction. The factor of 3.2 discrepancy between the two values listed for the optical nucleus is due to differences in the size of the region. While this work gives the value for the central $1^{\prime \prime} \times 1^{\prime \prime}$, the Kim, Veilleux, \& Sanders 1998 value corresponds to a region $2^{\prime \prime}$ wide along the east-west direction.

c The factor of almost 2 discrepancy between the two values for the optical nucleus is due to aperture effects (see above). The larger value represents a more reliable value, since it corresponds to a smaller aperture centered on the optical peak emission.

${ }^{\mathrm{d}}$ Extinction-corrected luminosity using the listed $A_{V}$ values in units of ergs s ${ }^{-1} \AA^{-1}$.

${ }^{\mathrm{e}}$ Emission-line ratios. The first row is for our measurements of the optical nucleus and presents the observed ratios, while the second row gives the extinction-corrected values. The values from Kim et al. are extinction-corrected with the value given in col. (2). The ratios for the near-IR nucleus have not been corrected for internal extinction. In addition to the hydrogen Balmer lines, the collisionally excited lines used are [O III] $5007 \AA$, [O I] $6300 \AA$, [N II] 6584 $\AA$, and the $[\mathrm{S}$ II] doublet $6717,6731 \AA$.

${ }^{f}$ All values taken from Kim et al. 1998, using a slit width of 2" along the east-west direction. According to Kim et al. 1995, the uncertainties in the line fluxes are between $5 \%$ and $10 \%$. 

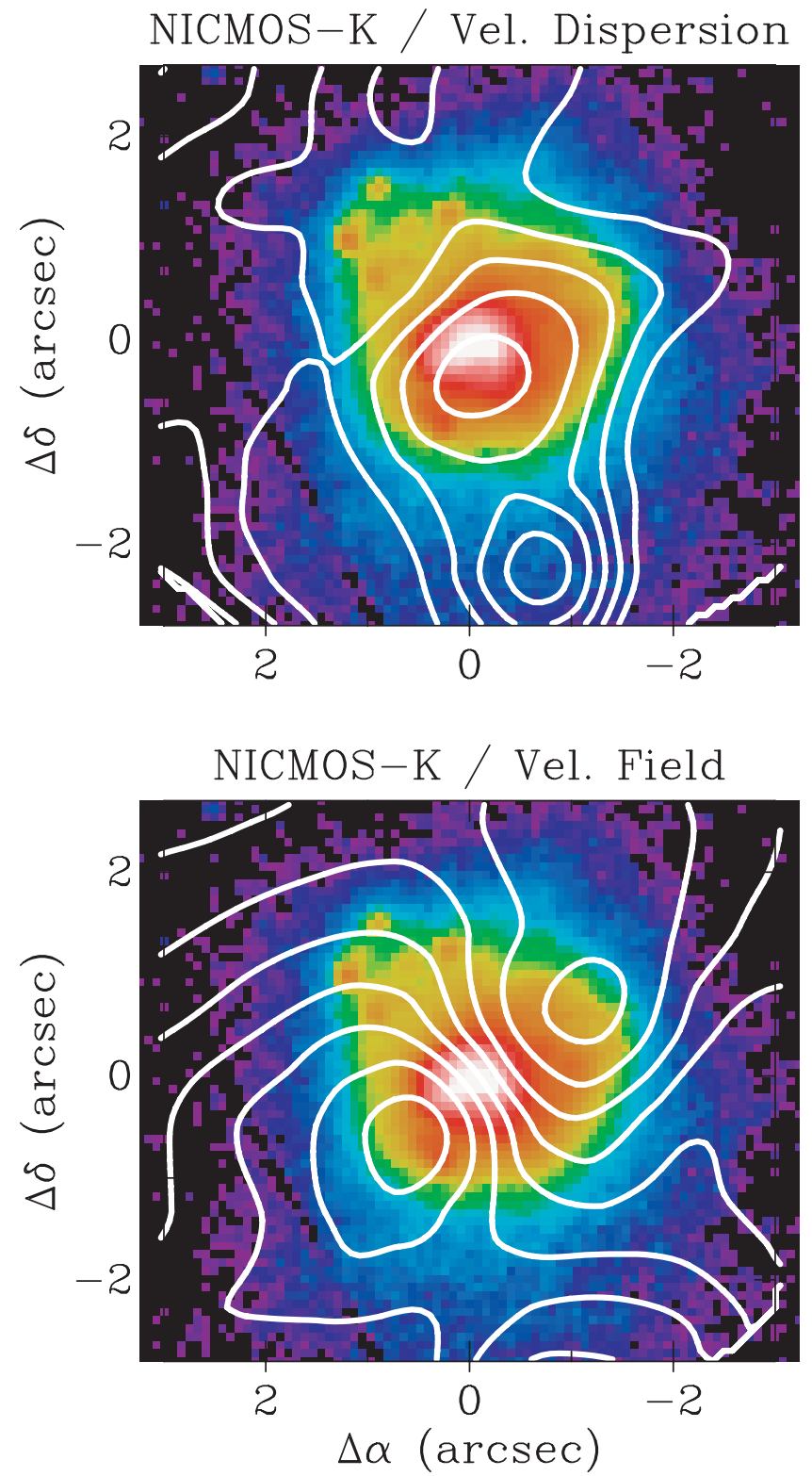

FIG. 3.-NICMOS $K$ image of IRAS 17208-0014 (first analyzed by Scoville et al. 2000), superposed on the INTEGRAL ionized gas velocity field (bottom) and the velocity dispersion map (top). The values for the isolines are (top) velocity field: 12,670, 12,711, 12,752, 12,794, 12,835, 12,877, 12,918 , and 12,960 $\mathrm{km} \mathrm{s}^{-1}$ (velocity field, bottom) and 240, 272, 304, 336, 368 , and $400 \mathrm{~km} \mathrm{~s}^{-1}$ (velocity dispersion map, top).

for instance, spectra 103 and 100). $\mathrm{H} \beta$ and [O $\mathrm{III}]$ are detected in only a few spectra outside the optical nucleus, with a ratio $[\mathrm{O} \mathrm{III}] / \mathrm{H} \beta \sim 0.5$. Note that for an $[\mathrm{O} \mathrm{III}] / \mathrm{H} \beta$ ratio lower than 1 , the values of the [O I] -, [N II]-, and [S II]to- $\mathrm{H} \alpha$ ratios, almost unaffected by internal extinction, are good discriminators between LINER and $\mathrm{H}$ II ionization (Veilleux \& Osterbrock 1987). Therefore, our new INTEGRAL spectroscopy shows that outside the optical nucleus, the excitation properties of the ionized gas are characteristic of LINERs (see Table 1 and Fig. 2). This includes the nearIR nucleus (i.e., the true nucleus; see $\S 3.4$ ), which is therefore classified as a LINER (Table 1). Since previous classifications were based on long-slit spectroscopy of the optical nucleus (Veilleux et al. 1995), the classification of the nucleus of IRAS 17208-0014 has to be changed to LINER. This new classification is not in contradiction with the classification as a starburst based on ISO spectroscopy (Lutz et al. 1999), especially if we take into account the differing spatial resolution. On the contrary, as pointed out by these authors, rather than indicating the presence of an active nucleus, the LINER spectra in ULIRGs are more likely due to shocks produced by compact and massive starbursts.

\subsection{Massive Extranuclear Starburst}

The properties of the optical emission peak, i.e., the optical nucleus, indicate that a massive, young starburst is taking place in an extranuclear region (see $\S 3.4$ ). The strong hydrogen recombination lines, with equivalent widths of $94 \pm 10$ and $13 \pm 3 \AA$ for $\mathrm{H} \alpha$ and $\mathrm{H} \beta$, respectively, indicate the presence of a young burst of about 5-6 Myr. Considering an age of $6 \mathrm{Myr}$, the dereddened $\mathrm{H} \alpha$ luminosity (see Table 1) and the WFPC2 I-band absolute magnitude ( $M_{I}=-21.7$, if $A_{V}=5.5 \mathrm{mag}$ is assumed) can be used to obtain two independent estimates of the total mass in the burst. Assuming the predicted $\mathrm{H} \alpha$ and $I$-band luminosity for an instantaneous burst characterized by a Salpeter initial mass function and stellar masses in the $1-100 M_{\odot}$ range (STARBURST99; Leitherer et al. 1999), the two estimates agree and give a total mass of $(3 \pm 1) \times 10^{8} M_{\odot}$. The bolometric luminosity of such a young and massive starburst is $(6 \pm 2) \times 10^{10} L_{\odot}$. Thus, although very massive, this starburst still produces a small fraction of the energy output of IRAS $17208-0014$, equivalent to $2.5 \%$ of its infrared luminosity. The region where this starburst is located has a size of about $0.9 \mathrm{kpc}$ in diameter. Therefore, it is likely that this starburst, as suggested by the HST images, does not form a single entity but is distributed in several less massive clusters. In this respect, most of the bright clusters identified in the near-IR are located in this region (Scoville et al. 2000). In particular, the two first-ranked $K$-band clusters alone (clusters 1 and 9 in Scoville et al. 2000) produce a combined bolometric luminosity of $2 \times 10^{10} L_{\odot}$, i.e.. a factor of 3 less than the luminosity of the starburst, if the same age and extinction as measured for the entire region are assumed.

\subsection{Kinematic Tracing of the Dust-enshrouded Dynamical Nucleus and Inner Gas Disk}

The velocity field of the ionized gas inferred from the $\mathrm{H} \alpha+[\mathrm{N}$ II] lines is shown in Figure 2 (bottom right-hand panel). The largest velocity gradient is found not across the optical nucleus but across the infrared nucleus (see also Fig. 3 , bottom). This indicates that the dynamical nucleus is in positional agreement with the infrared nucleus. Therefore, even if the nuclear power source is buried at near-IR wavelengths, the infrared nucleus indicates its direction. Note that the highly symmetric pattern of the ionized gas velocity field allows us to accurately locate the position of the true nucleus using optical spectroscopy (i.e., without the need of near-IR observations)

The peak-to-peak velocity difference corresponds to $\Delta v \sin i=250 \mathrm{~km} \mathrm{~s}^{-1}$ along P.A. $\sim 130^{\circ}$. This direction is in good agreement with that of the major photometric axis inferred from the inner isolines in the NICMOS images (excluding the effects from the clusters; see Fig. 3). The agreement between the photometric and kinematic axes suggests that we are observing a rotating disk of ionized gas. The kinematic axes of this disk are also in good positional 
agreement with those of the molecular (CO) disk found by Downes \& Solomon (1998). It is also worth noting that the velocity field does not show any evidence for the presence of a second nucleus.

Outside the inner disk the isovelocities twist, having a characteristic S-shape. Although this may indicate warps (see, e.g., Mediavilla, Arribas, \& Rasilla 1992), we believe that it is due to the transition from inner disk to outer envelope seen in projection, as suggested by fact that the isovelocity distortions appear at the edge of the inner disk.

The velocity dispersion map indicates relatively large velocity dispersions over most of the observed region (i.e., FWHM $\sim 300 \mathrm{~km} \mathrm{~s}^{-1}$ ). Within uncertainties, the infrared nucleus is in positional agreement with a local maximum $\left(\sim 450 \mathrm{~km} \mathrm{~s}^{-1}\right)$ in the velocity dispersion map (Fig. 3), which in turn is well centered with the kinematic center of rotation. Therefore, there is little doubt that the dynamical nucleus of the galaxy is located there. Another local maximum in the velocity dispersion map is observed toward the southwest, at about $3^{\prime \prime}(2.7 \mathrm{kpc})$. The mean velocity of this region is redshifted by about $200 \mathrm{~km} \mathrm{~s}^{-1}$ with respect to the systemic velocity, as can be observed in the velocity field map. These redshifted velocities are found in a position near the kinematic axis, where mainly systemic velocities are expected for a purely rotating disk. This indicates the presence of a kinematically distinct component.

\section{DISCUSSION}

\subsection{Tidally Induced Inflows}

In principle, the kinematically distinct component reported in $\S 3.4$ could indicate the presence of large-scale outflows originated by AGN-driven jets (Colbert et al. 1998 and references therein). However, the fact that no evidence for either an AGN or a jet has been reported for this object makes this explanation rather speculative. Superwinds (Heckman, Armus, \& Miley 1990) could also be, in principle, a possible explanation. However, if the spiral-like arms observed in optical images are trailing, the INTEGRAL velocity field of the inner disk (i.e., redshifted velocities in the southeast; blueshifted velocities in the northwest) indicates that the closer part of the disk is in the northeast. With this geometry, and assuming an inclination of $35^{\circ}$ (as suggested by the inner isolines in the NICMOS $K$ image), two outflowing components are expected to be seen in projection over the southwest and another two over the northeast (see the geometry described in Fig. 17 of Heckman et al. 1990). In the southwest, the component closest to us should have blueshifted velocities. The farthest component seen in projection over the southwest could have blueshifted or redshifted velocities, depending on the opening of the outflow cone. The fact that we only observe one extra component (out of four expected), which also has relatively strong redshifted velocities along the southwestern part of the minor axis, makes the superwind scenario very unlikely in the case of IRAS 17208-0014.

Alternatively, the kinematically distinct component could be associated with streaming motions of gas related to the merging process. The fact that these motions are found along the minor axis suggests that they are perpendicular to the disk of gas. Taking into account the geometry of the inner disk (i.e., with northeast being the closer part, $i=35^{\circ}$ ), the observed redshifted velocities along the south- western part of the rotation axis imply inflows. (Conversely, outflows along the southwestern part of the rotation axis would imply approaching velocities, i.e., a blueshifted component.) Note that the rotation axis is a canal where mass can travel efficiently toward the innermost parts of the galaxy without the limitations imposed by the conservation of angular momentum. In short, the extra redshifted component found along the southwestern part of the minor axis can be explained as the result of an inflow motion perpendicular to the disk. Even if the inflows may lose efficiency at the innermost regions (i.e., the inner kiloparsecs), their existence supports the idea that, as a consequence of the merging process, gas is channeling from the external regions, several kiloparsecs away, into the nuclear regions where the massive starburst described in $\S 3.3$ is taking place. The presence of strong inflows and associated star formation are expected during the final coalescence phase of two disk galaxies with bulges (Mihos \& Hernquist 1996). IRAS $15206+3342$ (Arribas \& Colina 2002) is another case in which these types of motions are clearly observed.

\subsection{IRAS 17208-0014 Near-IR Luminosity: Extinction Corrections for ULIRGS}

The measurements of the luminosity of ULIRGs in the near-IR and the internal extinction corrections to be applied are not well defined. Some authors (Genzel et al. 2001) argue that extinction over the entire size of these galaxies is very large and that therefore corrections to the integrated apparent magnitudes, even in the $K$ band, are not negligible; i.e., mean corrections of $0.7 \mathrm{mag}$ have to be applied. Others (Colina et al. 2001) indicate that dust distribution is very patchy in ULIRGs and that therefore, while internal extinction toward the nucleus can be high, the mean extinction over the entire ULIRG is much less as a result of large differential extinction gradients. In the following, we discuss the differential extinction in IRAS 17208-0014 and show how this extinction should be taken into account in order to obtain a reliable near-IR luminosity of this galaxy and of ULIRGs in general.

In IRAS 17208-0014, NICMOS colors (Scoville et al. 2000) and the INTEGRAL spectroscopy presented here (see $\S 3.1$ ) indicate visual extinctions of about 8 and $5.5 \mathrm{mag}$ toward the near-IR nucleus and the extranuclear starburst (i.e., the optical nucleus), respectively. However, the mean extinction across the entire galaxy appears to be much less, as indicated by the bluer near-IR colors (Scoville et al. 2000) when the size of the aperture is increased from a 1 "' 1 to an 11 ". 4 diameter (i.e., from 1 to $10 \mathrm{kpc}$ at the assumed distance for IRAS 17208-0014). Stellar populations cover a limited and well-defined range of near-IR colors, with almost constant $J-H$ of about +0.8 and $H-K$ of +0.35 for starbursts older than $10 \mathrm{Myr}$ and slightly bluer colors for younger starbursts (STARBURST99; Leitherer et al. 1999). Therefore, the observed, integrated colors can be used to estimate the mean extinction within the galaxy. For IRAS 17208-0014, the NICMOS $J-H$ and $H-K$ colors measured within an aperture of 11 ".4, or $10 \mathrm{kpc}$ (i.e., covering the field of view of our INTEGRAL spectra), are +1.3 and +0.6 , respectively, and therefore the mean extinction $\left(A_{V}\right)$ is about $2.5 \mathrm{mag}$, much less than for the nuclei. Therefore, the extreme extinctions measured in the nuclear regions $(\sim 1 \mathrm{kpc})$ through small apertures cannot be applied to the entire galaxy. 
This fact is obviously important when computing the absolute near-IR magnitudes of ULIRGs in general and of this galaxy in particular. The observed apparent $H$-band magnitude of 12.09 in a region $10 \mathrm{kpc}$ in diameter, i.e., including the nucleus and host (Scoville et al. 2000), corresponds to an absolute magnitude of $M_{H}=-24.22$ (i.e., an $L^{*}$ galaxy) if no reddening correction is applied. If the mean extinction of $A_{V}=2.5 \mathrm{mag}$ is considered for the entire galaxy, the corresponding luminosity of the system is $1.5 \mathrm{~L}^{*}$.

\subsection{Characteristics of the Merger}

The morphological and kinematical properties of IRAS 17208-0014 indicate that this system is in an advanced phase of the merging process between two gas-rich galaxies. The predicted strong inflow (and associated star formation) in this phase is well detected in the case of IRAS $17208-0014$, as we have discussed in $\S 4.1$ (and $\S 3.3$ ). Highresolution NICMOS images (Scoville et al. 2000; see also Fig. 3) and INTEGRAL two-dimensional kinematics do not show evidence for a second nucleus. The massive young clusters are preferentially located in the circumnuclear regions (i.e., inner $1-2 \mathrm{kpc}$ ), with no signs of star-forming regions along (the observed inner parts of) the tidal tails, like those found in dynamically younger systems where two distinct nuclei are still present (e.g., IRAS $12112+0305$ : Colina et al. 2000; IRAS 08572+3915: Arribas et al. 2000; IRAS 14348-1447: A. Monreal et al. 2003, in preparation). Further morphological characteristics, e.g., a $\sim r^{1 / 4}$ intensity profile in the near-IR (Scoville et al. 2000), also indicate that IRAS $17208-0014$ is indeed an advanced merger/elliptical galaxy in formation.

The fairly regular disk kinematics shown in our velocity field (as well as the good alignment of the kinematic and photometric axes) points to an unequal-mass merger, according to the numerical simulations by Bendo \& Barnes (2000) and Naab \& Burkert (2001). This is supported by recent measurements of the central $V_{\text {rot }} / \sigma$ ratio from stellar kinematic data (Genzel et al. 2001). The $V_{\text {rot }} / \sigma$ value of 0.48 indicates that the merger product is a relatively fast rotator and therefore that the mass ratio of the two progenitor galaxies involved in the merger should be $\sim 2: 1$. Moreover, the relatively long (though faint) tidal tails in IRAS 17208-0014 suggest that the encounter could have been prograde (Dubinski, Mihos, \& Hernquist 1999).

The estimated dereddened $H$-band luminosity of IRAS $17208-0014$ corresponds to $1.5 L^{*}$ (see $\S 4.2$ ). This result by itself does not imply that the system is the merger of two massive spiral galaxies (i.e., $\left.\geq m^{*}\right)$. In fact, young (6-10 Myr) starbursts have $H$-band $M / L$ ratios a factor of 50 lower than those of old $1 \mathrm{Gyr}$ stellar populations. In other words, the same $H$-band luminosity will be produced by an old bulge population and by a 50 times less massive young starburst. As an example, the massive extranuclear starburst of $3 \times 10^{8} M_{\odot}$ (see $\S 3.3$ ) alone produces an $H$-band luminosity of $0.1 L^{*}$. Moreover, a continuous $100 M_{\odot} \mathrm{yr}^{-1}$ star formation over 10-20 Myr, inferred to be happening in ULIRGs in general, produces an $L^{*}$ luminosity in the $H$ band. Recent stellar kinematical measurements support low $M / L$ values. In fact, IRAS $17208-0014$ is located near the region of the FP populated by intermediate-mass $\left(\sim L^{*}\right)$ elliptical galaxies, and its dynamical mass corresponds to $1.1 \times 10^{11} M_{\odot}$, or $0.8 m^{*}$, from stellar velocity dispersion measurements (Tacconi et al. 2002). Although the gas kinematics may not necessarily trace the dynamical mass, we note that the present gas velocity dispersions are only somewhat lower than the stellar values, which also suggests a mass lower than $m^{*}$ for the system. These results therefore support the scenario proposed by Colina et al. (2001), in which cool ULIRGs like IRAS 17208-0014 would be produced during the coalescing of two low-mass disk galaxies.

In summary, the kinematical, morphological, and photometric evidence presented here supports the idea that we are witnessing in IRAS 17208-0014 a luminous, cool ULIRG that is at the final coalescence phase of a system composed of two low-mass spiral galaxies (i.e., $\leq m^{*}$ ) with a mass ratio of $2: 1$, each consisting of a disk+bulge internal structure, that have been involved in a prograde encounter. This system will most likely not evolve into a luminous QSO (see next section), but into a fast-rotating elliptical $\left(\sim L^{*}\right)$.

\subsection{IRAS 17208-0014: A Cool ULIRG not Evolving into a Luminous $Q S O$}

According to the evolutionary scenario first proposed by Sanders et al. (1988), cool ULIRGs would evolve into warm ULIRGs and finally into QSOs. This scenario implies that cool ULIRGs should be found preferentially among galaxies in the initial and intermediate phases of the merging process, while the galaxies involved in the collision still preserve much of their individual identities or are separated enough that their nuclei have not merged yet. On the other hand, warm ULIRGs should be found in late phases and therefore preferentially among close pairs or even singlenucleus galaxies, while QSOs should be located exclusively in compact, single-nucleus systems.

According to this scenario, IRAS 17208-0014, which is at an advanced merging phase (see $\S 4.3$ ) and has an infrared luminosity of $L_{\mathrm{IR}}=10^{12.4} \mathrm{~L} \odot$ (i.e., one of the most luminous ULIRGs in the $1 \mathrm{Jy}$ sample), should be showing the characteristics of a warm, QSO-like ULIRG. However, quite to the contrary, IRAS $17208-0014$ shows no signs of the presence of a QSO: (1) with an $f_{25 \mu \mathrm{m}} / f_{60 \mu \mathrm{m}}$ ratio of 0.05 , it is one of the coldest ULIRGs; (2) its near- and mid-IR emission shows the same extended structure of about $2^{\prime \prime}$ (Scoville et al. 2000; Soifer et al. 2000), with no evidence of a luminous, pointlike, nuclear source; and (3) there is no trace of a (dominant) AGN component from either the optical (this work) or the near- (Goldader et al. 1995) and mid-IR (Lutz et al. 1998) spectroscopy, and not even from the hard X-ray (Risaliti et al. 2000). Moreover, the low $f_{25} \mu \mathrm{m} / f_{60 \mu \mathrm{m}}$ and $F_{\mathrm{HX}} / F_{\mathrm{IR}}\left(1.25 \times 10^{-4}\right)$ flux ratios are close to those of luminous infrared (i.e., $L_{\mathrm{IR}} \leq 10^{11}$ ) starbursts and unlike those of luminous infrared galaxies with a Seyfert 1 nucleus (about 2 and 0.1, respectively; see Fig. 5 in Risaliti et al. 2000). In addition, considering the dereddened $\mathrm{H} \alpha$ luminosity for the entire galaxy $\left(3.4 \times 10^{42} \mathrm{ergs} \mathrm{s}^{-1}\right)$, the ratio of hard X-ray to $\mathrm{H} \alpha$ luminosity is 0.35 , much less than the median value of 15 for QSOs, Seyfert 1, and low-luminosity LINER 1 nuclei (Ho et al. 2001) and similar to the average measured value (0.14) in nearby nuclear starbursts (Pérez-Olea \& Colina 1996). Finally, the low radio flux density of $102 \mathrm{mJy}$ at $1.4 \mathrm{GHz}$ (Condon et al. 1996) gives an $\mathrm{X}$-ray-to-radio luminosity ratio of 520, also in agreement with the average value of 400 in nearby nuclear starbursts (Pérez-Olea \& Colina 1996). 
In addition to these energy arguments, there are also dynamical arguments indicating that if IRAS 17208-0014 harbors a central black hole, it must have a relatively low mass and therefore not be capable of producing a luminous QSO $\left(M_{\mathrm{BH}} \sim 10^{9} M_{\odot}\right)$. The estimated mass of the IRAS 17208-0014 black hole is $2.3 \times 10^{8} M_{\odot}$, based on groundbased, near-IR stellar velocity dispersion measurements and assuming that the correlation between the black hole masses and bulge velocity dispersion for early-type galaxies also holds for late-phase ULIRGs (Tacconi et al. 2002). The stellar velocity dispersion has been measured over a region of about $1 \mathrm{kpc}$ in diameter $(0.5-1.2 \mathrm{kpc}$ effective radii, as mentioned in Tacconi et al. 2002). The central gas and stellar distributions in ULIRGs differ dramatically from those of elliptical galaxies and the bulges of spiral galaxies because of their large concentrations of high-density molecular gas and young, massive stellar clusters. Therefore, it is likely that in ULIRGs, aside from the black hole itself, these stellar and gas components make up a nonnegligible fraction of the total mass. Therefore, the mass estimate of the nuclear black hole mass should most likely be considered as an upper limit to the true mass of the black hole.

In summary, despite its advanced dynamical phase, starbursts seem to be the dominant energy source in IRAS 17208-0014, and if an AGN exists, it must produce only a minor contribution to the overall energy and ionizing budget. We note that similar characteristics (i.e., advanced merger without clear evidence of a QSO) have also been found in IRAS $15206+3342$ (Arribas \& Colina 2002). These cases do not follow the behavior expected in the "ULIRGto-QSO"'evolutionary scenario proposed by Sanders et al. (1988), therefore questioning its universality. On the other hand, if the alternative scenario proposed by Colina et al. (2001) is correct, IRAS 17208-0014 represents an example of the merger of two low-mass disk galaxies that will produce a luminous, cool ULIRG but will never evolve into a QSO phase.

\subsection{Implications for High-z Dust-enshrouded Massive Starburst Galaxies}

There are three fundamental questions that have to be answered in the future in order to advance our knowledge of high- $z$ galaxies: (1) what is the fraction of AGNs as a function of redshift? (2) what is the mass distribution of the pregalactic objects in the early universe? and (3) how do pregalactic objects evolve into present-day galaxies (i.e., interaction/merging rates and the number of colliding objects)? The results presented for IRAS 17208-0014 illustrate the problems and caveats that have to be taken into account when investigating high- $z$ dusty objects and before answering the above-mentioned questions.

IRAS $17208-0014$ is a clear example of a ULIRG in which the optical nucleus, in both continuum and emission lines, does not trace the true dynamical nucleus of the galaxy but is displaced by a large distance of $1.3 \mathrm{kpc}$. Moreover, IRAS 17208-0014 shows two strong continuum peaks in the optical that could naively be associated with the nuclei of two galaxies. However, two-dimensional kinematics and $K$-band imaging show that none of these optical nuclei are associated with the true single nucleus of this galaxy. Therefore, conclusions about the morphological properties and structure of the progenitors of high-z dusty starbursts, including merging rates, interaction phase, or number of colliding objects, would depend critically on a reliable twodimensional knowledge of the stellar structure and kinematical properties of the gas.

IRAS $17208-0014$ is also an example of misleading spectral classification of its nucleus in the optical. In the past, because of the lack of two-dimensional spectroscopy, this ULIRG has been classified as an $\mathrm{H}$ II, while the true nucleus has a LINER spectrum, as shown here. If this is a common situation, it could have severe implications. Since the fraction of AGNs in galaxies as a function of redshift can trace the formation and growth of massive black holes at early epochs, establishing a reliable classification of the nuclei of high- $z$ galaxies, in particular of the most common lowluminosity AGNs, is obviously needed.

Regarding its kinematics, the largest velocity gradient and the velocity dispersion peak in IRAS 17208-0014 are not found toward the optical nucleus, but toward the optically faint $K$-band nucleus. If the emission peak of the ionized gas is decoupled from the stellar continuum, or the stellar and gas distribution do not trace the true nucleus of the galaxy, any mass estimate from optical emission lines (e.g., $\mathrm{H} \alpha$ ) based on spatially unresolved velocity dispersion measurements will be affected by an undetermined error, depending on the specific object. IRAS 17208-0014 is not an isolated case among ULIRGs. Other ULIRGs for which IFS is available (i.e., Mrk 273: Colina, Arribas, \& Borne 1999; IRAS 12112+0305: Colina et al. 2000; IRAS 08572+3915: Arribas et al. 2000; Arp 220: Arribas, Colina, \& Clements 2001; IRAS 15206+3342: Arribas \& Colina 2002) do show similar features, most notably the decoupling between the stellar and ionized gas structure, offsets between the velocity dispersion peak and the optical light distribution, and strong differential extinction effects that could lead to wrong conclusions if investigated without adequate, spatially resolved, two-dimensional spectroscopy.

Future near- and mid-IR observations of high- $z$ dusty galaxies with large telescopes will effectively observe the rest-frame optical spectrum $(0.4-0.7 \mu \mathrm{m})$ for redshifts of 3 or larger. If, as demonstrated in this paper (see also Arribas \& Colina 2002), optical IFS data are relevant for adequately interpreting the properties of local ULIRGs, so should near- and mid-IR IFS be for interpreting high- $z$ galaxies. Although sensitivity and angular resolution (which worsen with wavelength) may limit these types of studies, a good understanding of the observational effects from analyzing a representative sample of local ULIRGs seems crucial in order to grasp the limitations of poorer linear resolution and one-dimensional studies.

\section{SUMMARY}

Integral field optical fiber spectroscopy with the INTEGRAL system, together with archival HST WFPC2 and NICMOS images, has been used to investigate the ULIRG IRAS 17208-0014. The main results are the following:

1. Differential extinction plays a major role in interpreting the innermost regions of this system. The optical nucleus (in both the continuum and line emission) is not coincident with the true (near-IR and dynamical) nucleus, but is displaced by $1.3 \mathrm{kpc}(1.5)$ from it. As a consequence, the previous optical spectral classification of the nucleus of this galaxy has to be changed from H II to LINER. 
2. A mean internal extinction equivalent to $2.5 \mathrm{mag}$ in the visual is derived for the galaxy. Because of the patchy structure of the dust distribution, this average extinction is much lower than the values (5.5-8 mag) measured in the nuclear regions. A dereddened $H$-band luminosity of $\sim 1.5 L^{*}$ is found for the entire system. Most of this luminosity is dominated by the young starbursts, not by the old bulge stellar population of the progenitor galaxies. In fact, in agreement with recent stellar measurements (Tacconi et al. 2002), our data suggest that IRAS 17208-0014 is a low-mass (i.e., $<m^{*}$ ) system.

3. The ionized gas emission is concentrated around the optical nucleus, where several luminous star-forming clusters are observed in the HST images. Our multiwavelength analysis indicates that the age of the burst is 5-6 Myr, its mass $(3 \pm 1) \times 10^{8} M_{\odot}$, and its bolometric luminosity $(6 \pm 2) \times 10^{10} L_{\odot}$. This starburst produces only a small fraction of the energy output of IRAS 17208-0014, equivalent to $2.5 \%$ of its infrared luminosity.

4. No strong line emission tracing star-forming regions or tidal dwarf galaxies is observed in the inner parts of the tidal tails, in contrast with that found in dynamically young double-nucleus ULIRGs. In fact, the secondary nucleus observed in the optical continuum (at $2.7 \mathrm{kpc}$ from the optical nucleus) vanishes at longer wavelengths and does not have distinct kinematics, excluding the possibility of its being associated with the nucleus of one of the merging galaxies.

5. The two-dimensional velocity field has identified the dynamical center of the galaxy as the region coincident with the near-IR nucleus detected in the NICMOS images. The velocity field also indicates that the $3 \mathrm{kpc}$ inner disk detected in the $K$ image has a regular rotation pattern $(\Delta v \sin i=250$ $\mathrm{km} \mathrm{s}^{-1}$ ). The kinematic and photometric axes are well aligned, which suggests that the stellar and gas components are coupled in this region. No kinematic evidence for a secondary nucleus is found.

6. Radial motions of gas are found along the minor kinematic axis. According to the geometry of the system (fixed by the velocity field and the assumption that the spiral armlike structures are trailing), the radial motions are well interpreted as inflows perpendicular to the inner disk. The existence of such inflows supports the idea that, as a consequence of the merging process, gas is channeling from the external regions, several kiloparsecs away, into the nuclear regions where the massive starburst mentioned above is taking place.

7. The kinematical, morphological, and photometric evidence presented here supports the idea that in IRAS 17208-0014 we are witnessing a luminous, cool ULIRG that is at the final coalescence phase of a system composed of two low-mass spiral galaxies (i.e., $\leq m^{*}$ ) with a mass ratio of $2: 1$, each consisting of a disk + bulge internal structure, that have been involved in a prograde encounter. This system will most likely evolve into a rotating, intermediatemass $\left(\sim L^{*}\right)$ elliptical galaxy.

8. All the multifrequency empirical evidence gathered so far indicates that IRAS 17207-0014 does not have a luminous QSO nucleus. Starbursts seem to be the dominant energy source, and therefore if an AGN exists, it must produce a minor contribution to the overall energy and ionizing budget. Therefore, IRAS 17208-0014 does not follow the behavior expected in the ULIRG-to-QSO evolutionary scenario proposed by Sanders et al. (1988). In fact, the present data (as well as those of IRAS 15206+3342; Arribas \& Colina 2002) are in better agreement with the alternative scenario proposed by Colina et al. (2001), according to which cool ULIRGs, such as IRAS 17208-0014, are the merger of two low-mass $\left(<m^{*}\right)$ disk galaxies that will never evolve into a QSO phase.

9. The present study illustrates some caveats to bear in mind when studying high- $z$ dusty galaxies without twodimensional spectral information at adequate linear resolution. Near- and mid-IR integral field spectroscopy is needed to investigate the nature and evolution of luminous infrared galaxies in the early universe.

We thank all the staff at the Spanish Observatorio del Roque de los Muchachos of the Instituto de Astrofísica de Canarias for their support. We appreciate the careful reading and comments from Ray Lucas, as well as those from an anonymous referee. Luis Colina thanks STScI for financial support under the Collaborative Visitors Program. We also thank Luis Cuesta for his help using GRAFICOS. Financial support for this work was provided by the Spanish Ministry for Science and Technology through grants PB98-0340C01, PB98-0340-C02, and AYA2002-01055.
Arribas, S., \& Colina, L. 2002, ApJ, 573, 576

Arribas, S., Colina, L., \& Borne, K. D. 2000, ApJ, 545, 228

Arribas, S., Colina, L., \& Clements, D. 2001, ApJ, 560, 160

Arribas, S., \& Mediavilla, E. 2000, in ASP Conf. Ser. 195, Imaging the Universe in Three Dimensions: Astrophysics with Advanced Multiwave-

length Imaging Devices, ed. W. van Breugel \& J. Bland-Hawthorn (San Francisco: ASP), 295

Arribas, S., et al. 1998, Proc. SPIE, 3355, 821

Bendo, G. J., \& Barnes, J. 2000, MNRAS, 316, 315

Bingham, R. G., Gellatly, D. W., Jenkins, C. R., \& Worswick, S. P. 1994, Proc. SPIE, 2198, 56

Colbert, E. J. M., Baum, S. A., O’Dea, C. P., \& Veilleux, S. 1998, ApJ, 496, 786

Colina, L., Arribas, S., \& Borne, K. D. 1999, ApJ, 527, L13

Colina, L., Arribas, S., Borne, K. D., \& Monreal, A. 2000, ApJ, 533, L9

Colina, L., et al. 2001, ApJ, 563, 546

Condon, J. J., Helou, G., Sanders, D. B., \& Soifer, B. T. 1996, ApJS, 103, 81

Downes, D., \& Solomon, P. M. 1998, ApJ, 507, 615

Dubinski, J., Mihos, J. C., \& Hernquist, L. 1999, ApJ, 526, 607

Duc, P.-A., Mirabel, I. F., \& Maza, J. 1997, A\&AS, 124, 533

Genzel, R., Tacconi, L. J., Rigopoulou, D., Lutz, D., \& Tecza, M. 2001, ApJ, 563, 527

\section{REFERENCES}

Genzel, R., et al. 1998, ApJ, 498, 579

Goldader, J. D., Joseph, R. D., Doyon, R., \& Sanders, D. B. 1995, ApJ, 444, 97

Heckman, T. M., Armus, L., \& Miley, G. K. 1990, ApJS, 74, 833

Ho, L. C., et al. 2001, ApJ, 549, L51

Howarth, I. D., \& Murray, J. 1988, DIPSO: A Friendly Spectrum Analysis Program (Starlink User Note 50; Chilton: Rutherford Appleton Lab.)

Kim, D.-C., Sanders, D. B., Veilleux, S., Mazzarella, J. M., \& Soifer, B. T. 1995, ApJS, 98, 129

Kim, D.-C., Veilleux, S., \& Sanders, D. B. 1998, ApJ, 508, 627 2002, ApJS, 143, 277

Leitherer, C., et al. 1999, ApJS, 123, 3

Lutz, D., Spoon, H. W. W., Rigopoulou, D., Moorwood, A. F. M., \& Genzel, R. 1998, ApJ, 505, L103

Lutz, D., Veilleux, S., \& Genzel, R. 1999, ApJ, 517, L13

Martin, J. M., Bottinelli, L., Dennefeld, M., Gouguenheim, L., \& Le Squeren, A. M. 1989, A\&A, 208, 39

Mediavilla, E., Arribas, S., \& Rasilla, J. L. 1992, ApJ, 396, 517

Mihos, J. C., \& Bothun, G. D. 1998, ApJ, 500, 619

Mihos, J. C., \& Hernquist, L. 1996, ApJ, 464, 641

Murphy, T. W., Jr., Armus, L., Matthews, K., Soifer, B. T., Mazzarella, J. M., Shupe, D. L., Strauss, M. A., \& Neugebauer, G. 1996, AJ, 111, 1025 
Murphy, T. W., Jr., Soifer, B. T., Matthews, K., Armus, L., \& Kiger, J. R. 2001, AJ, 121, 97

Naab, T., \& Burkert, A. 2001, in ASP Conf. Ser. 230, Galaxy Disks and Disk Galaxies, ed. J. G. Funes \& E. M. Corsini (San Francisco: ASP), 451

Pérez-Olea, D. E., \& Colina, L. 1996, ApJ, 468, 191

Rieke, G. H., \& Lebofsky, M. J. 1985, ApJ, 288, 618

Risaliti, G., Gilli, R., Maiolino, R., \& Salvati, M. 2000, A\&A, 357, 13

Sanders, D. B., \& Mirabel, I. F. 1996, ARA\&A, 34, 749

Sanders, D. B., Soifer, B. T., Elias, J. H., Neugebauer, G., \& Matthews, K. 1988, ApJ, 328, L35

Scoville, N. Z., et al. 2000, AJ, 119, 991
Soifer, B. T., et al. 2000, AJ, 119, 509

Surace, J. A., Sanders, D. B., Vacca, W. D., Veilleux, S., \& Mazzarella, J. M. 1998, ApJ, 492, 116

Tacconi, L. J., Genzel, R., Lutz, D., Rigopoulou, D., Baker, A. J., Iserlohe, C., \& Tecza, M. 2002, ApJ, 580, 73

Veilleux, S., Kim, D.-C., \& Sanders, D. B. 1999, ApJ, 522, 113

- 2002, ApJS, 143, 315

Veilleux, S., Kim, D.-C., Sanders, D. B., Mazzarella, J. M., \& Soifer, B. T. 1995, ApJS, 98, 171

Veilleux, S., \& Osterbrock, D. E. 1987, ApJS, 63, 295

Wilman, R. J., Crawford, C. S., \& Abraham, R. G. 1999, MNRAS, 309, 299 